\title{
THE CONDUCTING SYSTEM OF THE VERTEBRATE HEART*
}

\author{
BY \\ FRANCIS DAVIES \\ From the Department of Anatomy, University of Sheffield \\ Received April 24, 1942
}

A study of the comparative anatomy of the conducting system of the vertebrate heart reveals that the phylogenetic evolution of this system affords a further illustration of the dictum on the close correlation between form and function enunciated by John Hunter. My present thesis is this. A cardiac conducting system, consisting of specialized muscle (i.e. nodal tissue and Purkinje fibres), is limited to mammals and birds. The general topography of this system is closely similar in these two classes of warm-blooded vertebrates; the main differences can be correlated with different functional requirements. This system is a neomorphic development; it is not a "remnant" of more extensive tissues of similar structure in lower vertebrate hearts. The system has undergone parallel evolution in mammals and birds in response to functional requirements. The evidence in support of this thesis may be briefly summarized as follows.

\section{MAMMALS}

The sinu-atrial (S-A) node, the site of initiation of the impulse for cardiac contraction (pace-maker), was originally described by Keith and Flack as lying superficially beneath the epicardium in the upper part of the sulcus terminalis, adjacent to the end of the superior vena cava. Subsequent studies have shown that the node is a much more extensive structure, being horse-shoe shaped, and embracing the ventral aspect of the end of the superior caval vein, the anterior limb of the horse-shoe extending caudally over more than the upper half of the sulcus terminalis (Fig. 1). Moreover, the node extends through the entire thickness of the atrial wall, from epicardium to endocardium. Some investigators have claimed that, after removal of the entire node, normal cardiac rhythm is maintained; they have therefore postulated the existence of other (ectopic) centres in the atria with the same rhythmic properties as the node. Owing to the difficulty of complete removal of this extensive node, and since even a small portion of nodal tissue left behind will be capable of initiating atrial contraction, the validity of such experimental work, not subejcted to strict histological control, is open to serious question.

* Abridged form of the Hunterian Lecture delivered at the Royal College of Surgeons of England, February 9, 1942. 


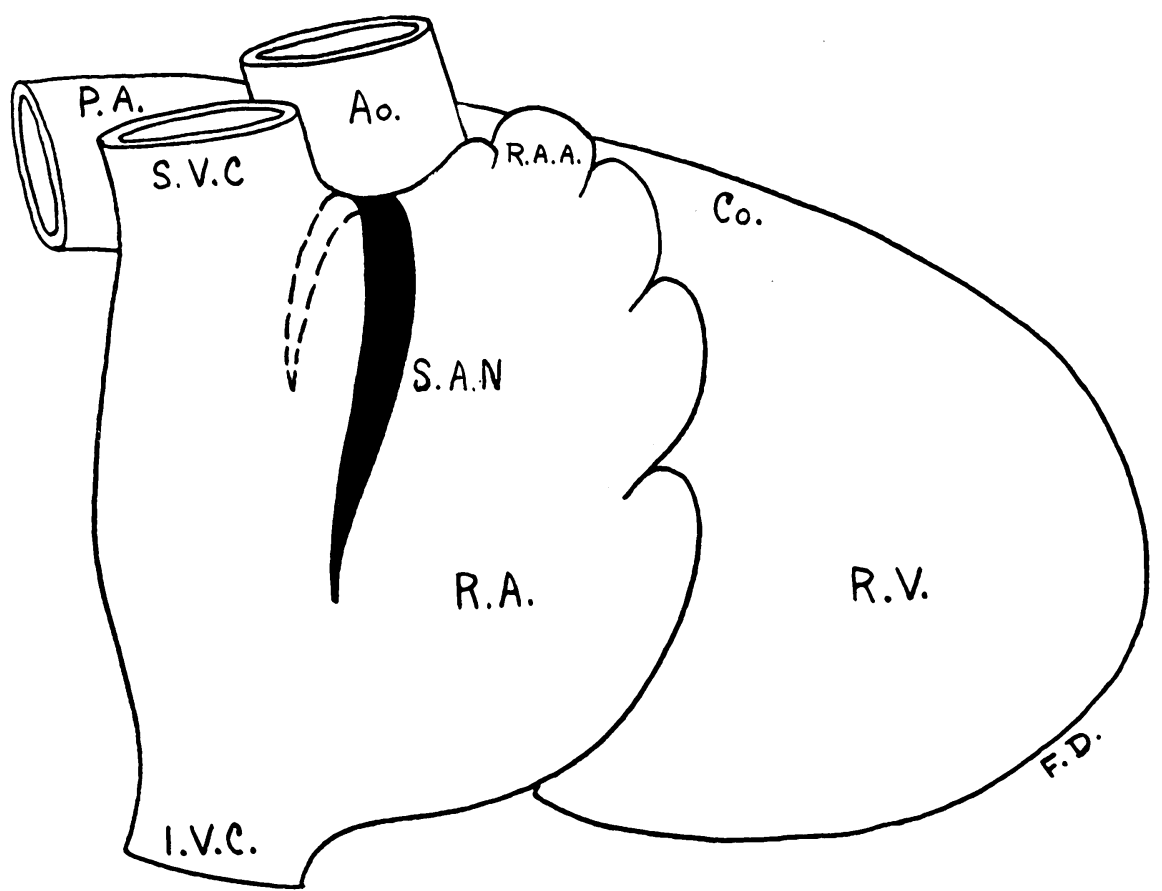

FIG. 1.-Extent of the S-A node in the mammal.

Ao., aorta; Co., conus arteriosus; I.V.C., inferior vena cava; P.A., pulmonary artery; R.A., right atrium; R.A.A., right atrial appendix; R.V., right ventricle; S.A.N., sinuatrial node; S.V.C., superior vena cava.

The node was described by its discoverers as a plexiform mass of peculiar pale-staining fibres intermingled with connective tissue and nerves; they considered this tissue to be intermediate in structure between nerve and muscle, and therefore gave it the non-committal name of " nodal tissue." Silver impregnation methods clearly reveal, however, that the nodal fibres are specialized cardiac muscle fibres; they are completely cross-striated, fusiform, and branched, their chief distinguishing character being their small breadth in comparison with the ordinary atrial muscle. This is one of the features to be correlated with the special properties of the node, namely its greater rhythmicity and slower rate of conduction than ordinary heart muscle. These nodal fibres are directly continuous with the neighbouring myocardial fibres of the right atrium; the latter are continuous with those of the left atrium; the cardiac impulse is thus transmitted through the atria by the ordinary cardiac muscle and reaches the atrio-ventricular node, whose specialized fibres are continuous with the myocardial fibres of both atria in the atrial septum.

A few observers have described a direct connection, by means of nodal tissue or Purkinje fibres, between the S-A and A-V nodes; most workers have failed to find such. Many investigators have noted the presence of typical large Purkinje fibres beneath the endocardium in both atria. From their descriptions these appear to be isolated collections of Purkinje fibres, unconnected with either the S-A or A-V node; moreover, the sites at which they have been found 
vary with the observers. We ourselves have seen a small collection of Purkinje fibres beneath the epicardium in the right atrium of the ox heart, which was continuous with the caudal end of the S-A node. It is probable that these conditions should be regarded as "variations," such as would be prone to occur in a newly evolved system.

The atrio-ventricular (A-V) node, smaller than the S-A node, is situated in the lower and dorsal part of the atrial septum, with whose musculature the special nodal fibres are continuous. The node is directly continued into the A-V bundle, which runs along the upper border of the ventricular septum to the lower edge of the pars membranacea septi, and there bifurcates into right and left limbs, the bifurcation straddling the upper border of the muscular part of the septum. The right limb runs down, in the form of a round bundle, on the right side of the septum, covered over by the endocardium and a thin layer of septal myocardium, making for the septal attachment of the " moderator band." The latter in most cases conveys the right limb across the ventricular cavity to the base of the anterior papillary muscle, where the limb divides into a plexus of Purkinje fibres which ramify beneath the endocardium throughout most of the right ventricle. Very rarely, the right limb passes from the septum to the opposite wall of the ventricle independently of the " moderator band," a feature that may be attributable to variation. The left limb, in the form of a broad flattened band, descends beneath the endocardium on the left side of the septum and, at a varying distance down the septum, divides into two or more branches, each of which, after a short course down the septum beneath the endocardium, subdivides into a number of branches. These latter are conveyed by the trabeculæ carneæ and the "false tendons" across the cavity to the papillary muscles on the opposite wall, there dividing into a plexus of Purkinje fibres which ramify beneath the endocardium throughout most of the left ventricle.

The A-V node, the A-V bundle and its limbs, together with the subendocardial Purkinje plexuses are surrounded by a fibrous sheath, which both mechanically protects the relatively (but not absolutely) stationary specialized elements from friction against the vigorously contracting ventricular myocardium, and also insulates them from the neighbouring cardiac muscle. Into the potential space between the sheath and the specialized muscle, fluids like indian ink can be injected, and the whole ventricular ramifications of this system can thus be readily and rapidly displayed. This method cannot be successfully employed in the human, because the sheath is extremely thin and ruptures with very slight increase in pressure, allowing the injection fluid to diffuse beneath the endocardium. Until recently, the subendocardial Purkinje fibres were thought to become continuous with the ventricular muscle immediately adjacent, which latter would thus convey the cardiac impulse through the thickness of the ventricular walls: but they themselves have now been shown (in a number of mammals) to penetrate throughout the entire thickness of the ventricular walls, including the septum, surrounded by their fibrous sheath, and to become continuous with the ordinary muscle at various depths from the surface; many of them penetrate the walls in relation to the branches of the coronary arteries. 
It may be observed that this discovery was made subsequent to the demonstration of a similar, but more widespread, penetration of Purkinje fibres in both ventricles and atria in the bird's heart. In the human, the ventricular Purkinje fibres are limited to the subendocardial plane. The Purkinje fibres that penetrate into the ventricular septum from the subendocardial plexuses on either side, communicate in the substance of the septum. Thus after damage of the upper part of the right limb of the A-V bundle for instance, the impulse can pass down the intact left limb, through these communicating septal Purkinje fibres to the right ventricle, reaching the latter after a delay that is very slightly greater than normal.

The discoverers of the S-A node remarked on its close similarity in histological structure to that of the A-V node, and it appears to have been generally accepted that the two nodes are identical in structure. The specialized muscle fibres of the A-V node, however, while completely crossstriated and more slender than the ordinary heart muscle, differ from those of the S-A node in being more cylindrical, more branched, and broader. These are some of the differences, to which may be attributed a probable lower rhythmicity than the S-A node and the fact that the cardiac impulse normally originates in the $\mathrm{S}-\mathrm{A}$ rather than the $\mathrm{A}-\mathrm{V}$ node.

In most mammals the A-V bundle and its two limbs consist of typical large Purkinje fibres. In the human, however, the fibres of the A-V bundle and of the proximal parts of its two limbs closely resemble the ordinary ventricular muscle fibres, as revealed by ordinary laboratory stains; in fact, the bundle fibres are slightly narrower than the myocardial fibres, and it is not until some distance down the septum that the typical large Purkinje fibres make their first appearance in the limbs. The pathologist may have difficulty in identifying these structures in isolated sections of the human heart; tracing continuity in serial sections is the only certain means of identifying them. The nodes, bundle, limbs, and terminal Purkinje fibres are accompanied by nerves, and numerous nerve cells are closely related to the nodes and bundle; these neural elements are capable of altering the degree of activity of the specialized conducting muscle under varying conditions. Although Kent described " accessory muscular connections" between the atria and ventricles in some mammals (rat and young rabbit; to a lesser extent, guinea-pig and hedgehog; very few in monkeys), the general opinion is that the A-V bundle constitutes the sole muscular $\mathrm{A}-\mathrm{V}$ connection in higher mammals.

\section{BIRDS}

The bird's heart resembles that of the mammal in being four-chambered, with complete atrial and ventricular septa. The heart rate, however, is considerably faster than that of a mammal of approximately the same size, and the necessity arises therefore for a more rapid spread of the cardiac impulse throughout the entire avian heart. Although Keith, Mackenzie, and others denied the existence of specialized nodes and bundle in the bird's heart, it has been shown (Davies) that not only are such structures present, but that the specialized conducting muscle is more widely distributed in the avian than in 


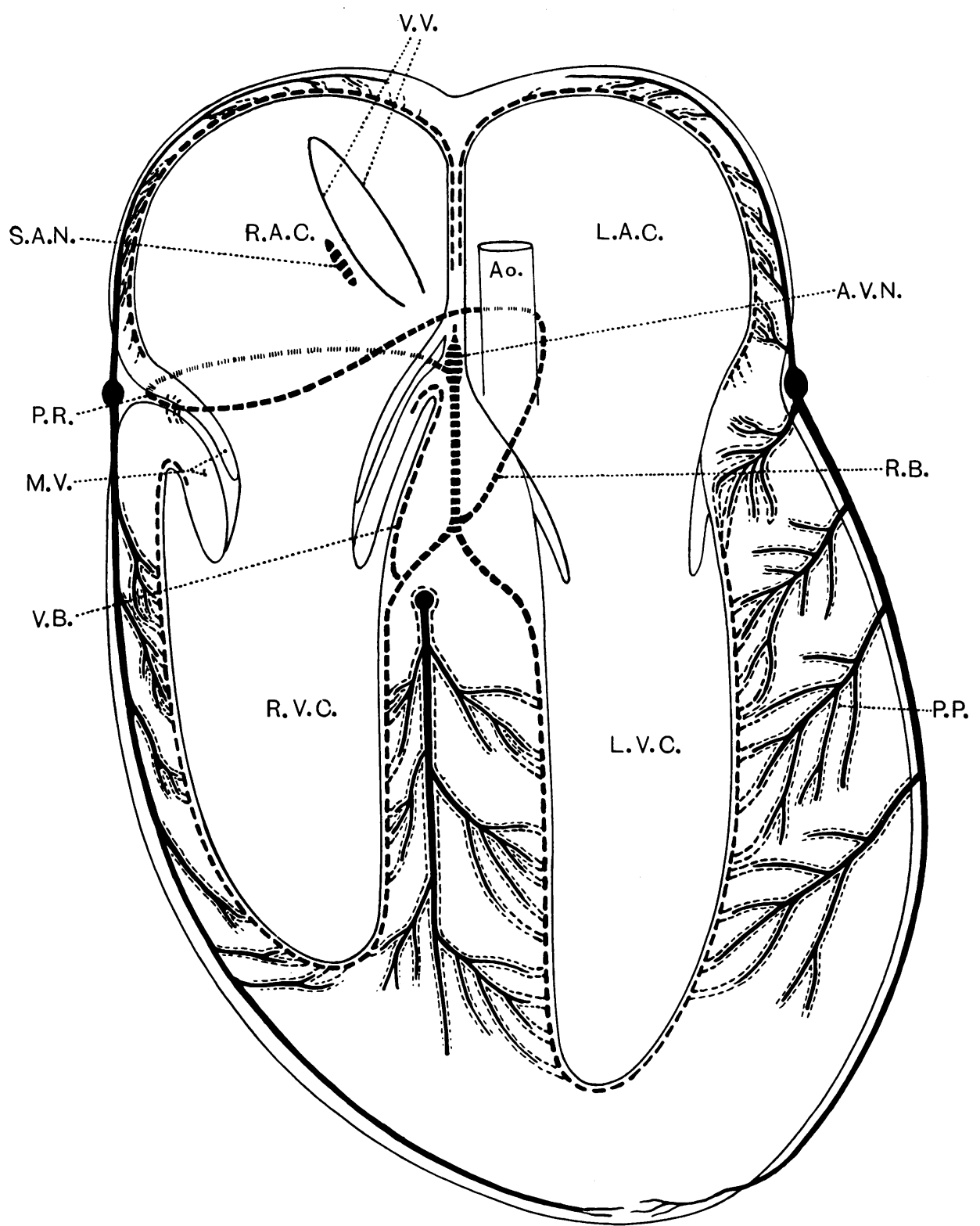

Fig. 2.-Diagram summarizing the main topographical disposition of the avian conducting system. For abbreviations see Fig. 3.*

the mammalian heart, and this latter is to be correlated with the need for more rapid diffusion of the impulse, as indicated above.

The S-A node, similar in structure to that of the mammal, lies on the right side of the entry of the right anterior vena cava into the right atrium, and extends throughout the entire thickness of the atrial wall. Unlike the mammal, however,

* I am indebted to the Editors of the Journal of Anatomy and to the Council of the Royal Society for permission to reproduce Fig. 2 and 3 respectively. 
the nodal fibres are continuous with large Purkinje fibres, which ramify beneath the endocardium throughout most parts of the right and left atria, and penetrate the atrial walls to become continuous with the ordinary atrial muscle, but are absent from the lower part of the atrial septum, so that, as in the mammal, the specialized fibres of the A-V node establish continuity with the ordinary cardiac muscle of the atrial septum.

The A-V node is similar in structure and position to that of the mammal and is continued directly into the A-V bundle. The latter, unlike the mammalian bundle, runs caudally and ventrally into the depth of the ventricular septum, lying approximately midway between its right and left surfaces. About one quarter of the way down the septum, the bundle divides into right and left limbs; these approach the corresponding sides of the septum and there divide into a plexus of Purkinje fibres, which ramify beneath the endocardium throughout most of the ventricular chambers, also penetrating through the thickness of the ventricular walls, mostly in relation to the coronary vessels, and becoming continuous with the ordinary ventricular muscle at varying depths from the surface. In the septum, as in the mammal, communications are established between the Purkinje fibres derived from the subendocardial plexuses on either side.

The difference in position of the mammalian and avian bundle in relation to the ventricular septum can be explained by the difference in the morphology of the septum in these two classes of warm-blooded vertebrates. The A-V bundle and its two limbs in the avian heart consist of large typical Purkinje fibres. A striking example of the effect that functional requirement exerts on the topographical distribution of the conducting elements is seen in relation to the right $\mathrm{A}-\mathrm{V}$ valve in the bird. In the mammal the tricuspid valve is a fibrous structure and closes passively by the pressure of the blood on its under surface during ventricular systole. In the bird this valve is a strong muscular structure, consisting mainly of an inflection of the base of the ventricle, covered on its inner surface by a thin prolongation of the atrial muscle, the epicardial connective tissue in the coronary sulcus being invaginated between the two. It is necessary that this muscular valve should contract actively at the outset of ventricular systole, and to this end early outgoing branches of the conducting system to the valve are present in the form of the right A-V ring of Purkinje fibres from the A-V node, which runs partly round the A-V orifice in the connective tissue of the valve, and an early branch from the right limb of the A-V bundle (see Fig. 2).

In the main then, the specialized conducting systems of mammalian and avian hearts are closely similar; differences can be correlated with different functional requirements.

\section{Lower Vertebrates and the Phylogeny of the Conducting Systems of Mammalian and Avian Hearts}

A number of workers, in particular Keith and Mackenzie, maintain that the nodes and A-V bundle of the mammal are remnants of more extensive tissues of similar structure in lower vertebrates. Thus, in the heart of the fish they 
describe a ring of nodal tissue at the junction of sinus venosus and atrium, in the base of the venous valves, and a similar ring at the junction of atrium and ventricle. To these nodal rings are attributed a greater rhythmicity and a slower rate of conduction of the cardiac impulse than the ordinary cardiac muscle; the latter property being responsible for the delay in the passage of the impulse from one cardiac chamber to the next: this delay is necessary in order that the chamber receiving blood may become completely filled before it in turn begins to contract. Keith and Mackenzie maintain that as one ascends the scale of animals the concentration and reduction of this tissue becomes more marked. Such concentration is already present in amphibia and becomes more marked in reptiles, in which the S-A ring is reduced to a collection of nodal tissue on the left side of the sinu-atrial junction. In mammals, associated with the development of fibrous rings separating the atrial from the ventricular musculature, the A-V ring of nodal tissue is reduced to the A-V node and A-V bundle, while the S-A nodal ring is concentrated to the S-A node.

Recent detailed examination of the heart of the spotted salamander (Davies and Francis) has thrown doubt upon this interpretation of the phylogenetic origin of the specialized conducting tissue of the mammalian (and avian) heart. This animal, a caudate amphibian, has a very primitive form of heart (Fig. 3), suitable for comparison with those of fish on the one hand and those of reptiles, birds, and mammals on the other. In it the four primary chambers (sinus, atria, ventricle, and bulbus) are well defined separate structures. No specialized muscle (i.e. nodal tissue or Purkinje fibres) is present in any part of this heart, either in the walls of the chambers or at the sites of junction of the several chambers. The cardiac muscle has the same histological structure throughout, as revealed by staining with hæmalum and eosin, Van Gieson and iron hæmatoxylin, and with Bodian activated protargol techniques. The arrangement of the muscle fibres, however, is noteworthy. With the exception of the bulbus, in which the cardiac muscle is circular throughout, the musculature of the chambers is arranged in a basket-work fashion.

At the sinu-atrial, atrio-ventricular, and ventriculo-bulbar junctions, however, the muscle fibres become abruptly changed to a circular arrangement, the sudden change in direction being a striking feature at each of the junctional sites. In each of these rings (of ordinary cardiac muscle) the fibres branch, the branches connect with the adjacent fibres, and this has the effect of converting these morphological rings into physiological spirals. The time taken by the cardiac impulse to traverse these spirals will, we believe, account for the delay in the passage of the impulse from one chamber to the next, without postulating the existence of special nodal (or block) fibres at these junctional sites. The analysis of superimposed tracings of the outlines of the heart of the salamander, made from slow-motion cinephotographic records of the normally beating heart, enables the course of the wave of contraction and the phases of a complete cardiac cycle to be determined. Such records indicate that the phases of the cardiac cycle in the heart of the salamander are similar to those of the mammalian heart. While similar detailed study of the hearts of fish (eel, gurnard), amphibia (frog) and reptiles (tortoise, Mississippi alligator, Sphenodon) still 


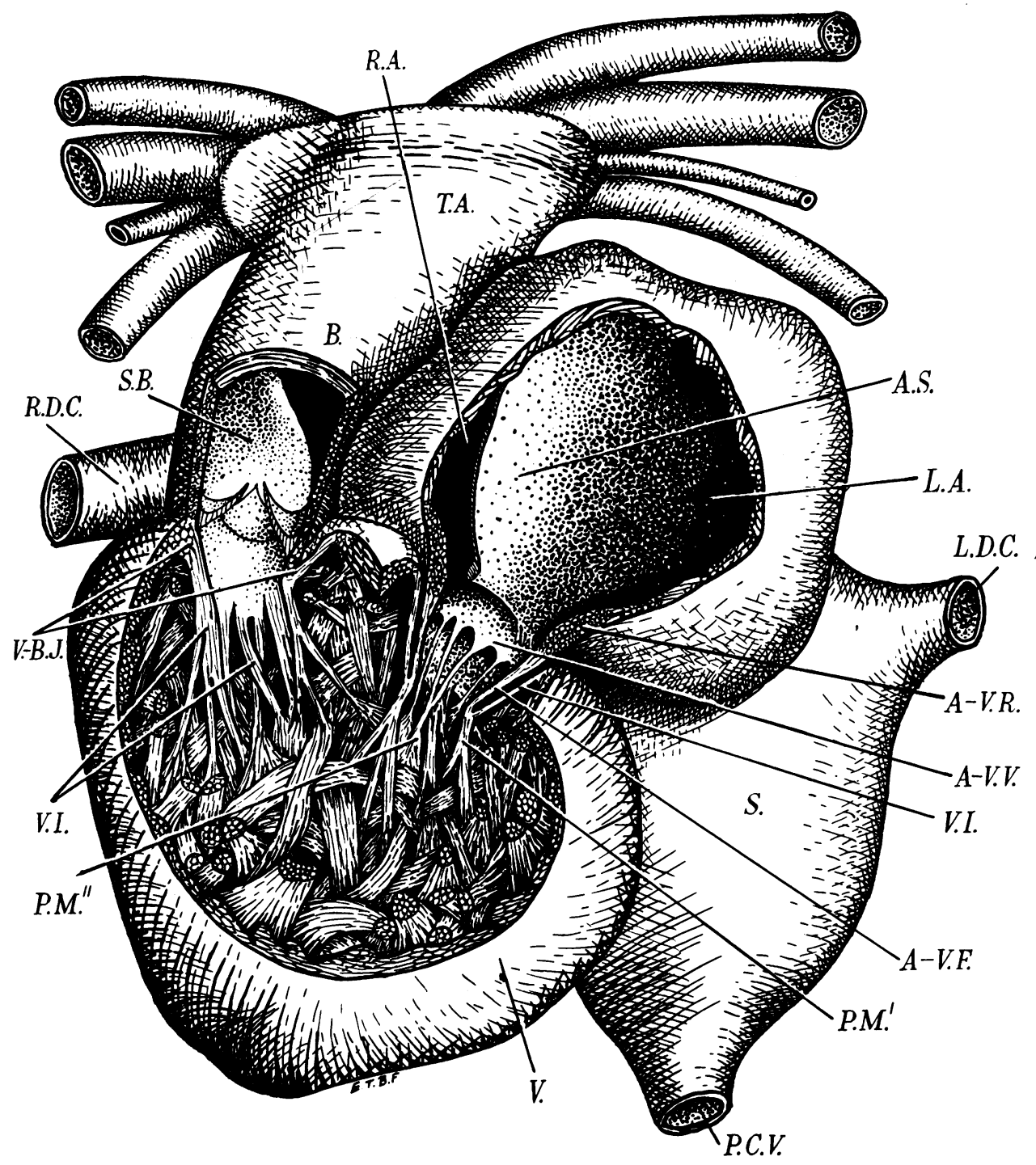

FIG. 3.-Dissection of the salamander's heart, showing atrio-ventricular and ventriculo-bulbar junctions.

A., atria; Ao., aorta; A.S., atrial septum; A-V.F., atrio-ventricular funnel; A-V.J., atrioventricular junction; A-V.N., atrio-ventricular node; A-V.R., atrio-ventricular ring; A-V.V., atrio-ventricular valve; B., bulbus; Co., conus arteriosus; I.V.C., inferior vena cava; L.A., left atrium; L.A.C., left atrial cavity; L.D.C., left duct of Cuvier; L.V.C., left ventricular cavity; M.V., muscular valve; P.A., pulmonary artery; P.C.V., postcaval vein; P.M.' and P.M.", papillary muscles; P.P.F., penetrating Purkinje fibres; P.R., right A-V Purkinje ring; R.A., right atrium; R.A.A., right atrial appendix; R.A.C., right atrial cavity; R.B., recurrent branch of $A-V$ bundle; R.D.C., right duct of Cuvier ; R.V., right ventricle ; R.V.C., right ventricular cavity ; S, sinus; S-A.J., sinu-atrial junction ; S-A.N., sinu-atrial node ; S.B., septum bulbi ; S.V.C., superior vena cava; T.A., truncus arteriosus ; V., ventricle ; V.B., branch of right limb of A-V bundle to muscular valve; V-B.J., ventriculo-bulbar junction; V.T., ventricular trabeculæ; V.V., venous valves. (Abbreviations for Fig. 2, 3 and 4). 
await completion, preliminary inspection of sections of these has failed to reveal the presence of nodal tissue or Purkinje fibres in any part of these hearts.

The conclusion is thus reached that the nodes and A-V bundle (together with its two limbs and the terminal subendocardial and penetrating Purkinje fibres) in the hearts of mammals and birds are neomorphic developments, which have undergone parallel evolution in association with the more rapid rate of the heart in proportion to its size, in these homoiothermal vertebrates. The heart rates of the frog, mouse, and canary are roughly 30 (at $15^{\circ} \mathrm{C}$.), 700 , and 1000 beats a minute respectively. These hearts are approximately of the same size and the cardiac impulse has therefore to be propagated throughout the heart more rapidly in the mouse than the frog, and more rapidly still in the canary. It might be expected that, whereas the ordinary cardiac muscle may be capable of conducting the impulse rapidly enough in the frog, special tissue which can conduct the impulse much more rapidly might be developed in the mouse and canary, and that this tissue would probably have a more extensive distribution in the bird than in the mammal. Such has been shown above to be the case.

When the sinus, atria, ventricle, and bulbus of the frog's heart are separated from each other by cuts made accurately through the junctions of these chambers, it is found that rate of rhythmic contraction of these chambers diminishes progressively from sinus to bulbus. In the absence of histologically specialized muscle, no satisfactory explanation of this difference in the intrinsic rhythmic rates of the several cardiac chambers has been advanced. Recent estimations of the relative glycogen content of the muscle fibres of these chambers have been made by staining sections with carmine, tracing the outline of the (highly magnified) fibres on squared paper and plotting in the redstained glycogen. The glycogen is found to increase progressively from sinus to bulbus (Davies and Francis, 1941) (Fig. 4). This order of increase is the same as that of the density and compactness of the cardiac musculature and of the work done by the several chambers, so that a positive correlation in this respect may be made, the glycogen possibly being a source of energy for the work of contraction. But the intrinsic rhythmicity diminishes in this order; whether this relation is merely coincidence or indicates a causal relationship remains to be determined. In the mammal, the nodes are particularly poor and the Purkinje fibres rich in glycogen in comparison with the ordinary cardiac muscle. The glycogen content of the muscle fibres at the S-A and A-V junctions of the frog's heart is intermediate between that of the muscle fibres of the adjacent chambers, and this may be taken as collateral supporting evidence that this junctional muscle is not the same as the nodal tissue or Purkinje fibres of the mammal.

Further collateral evidence that the nodes and bundle of the mammal are newly evolved structures is afforded by a study of their ontogeny in the calf heart. Shaner found that, in the $9 \mathrm{~mm}$. calf embryo, the A-V node appears behind the dorsal endocardial cushion and from it the A-V bundle grows on the top of the ventricular septum. At this time the developing musculatures of the atria and ventricles are continuous round the coronary sulcus. The A-V node and bundle thus appear and grow like any other embryonic organ 


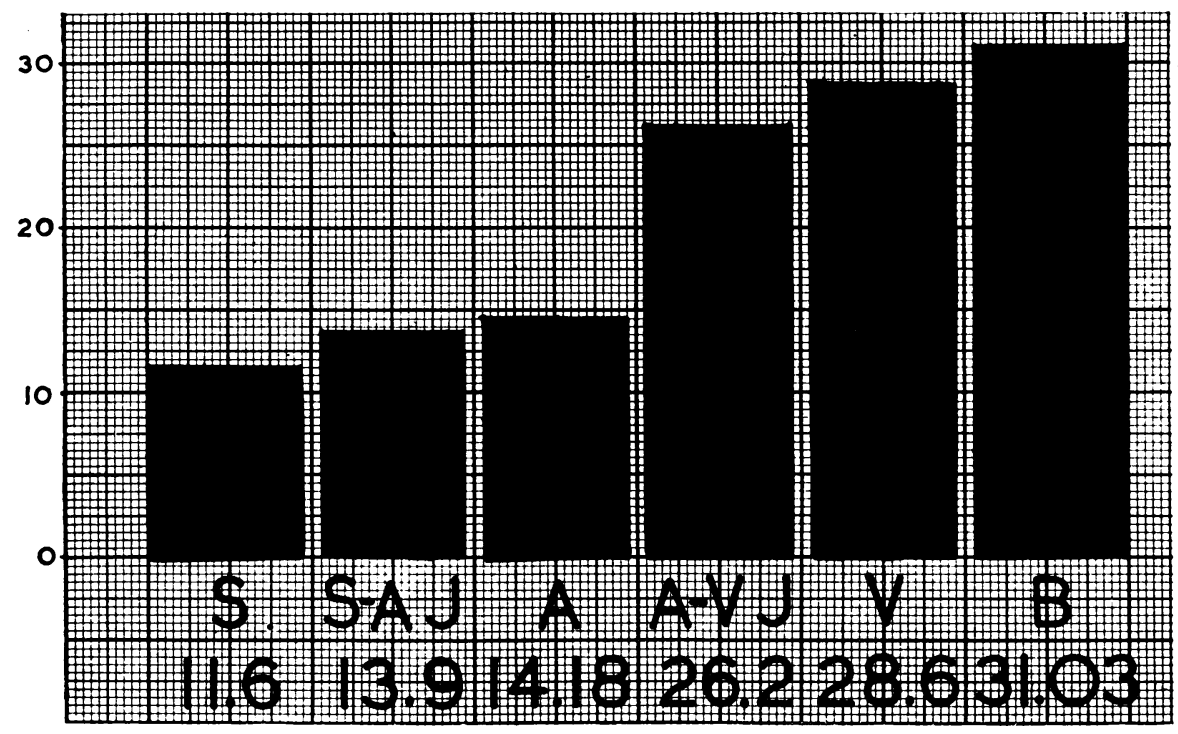

FIG. 4.-Glycogen content of the frog's heart. The relative amounts of glycogen in the muscle fibres of the cardiac chambers has been estimated graphically from sections stained by Best's alkaline carmine.

and are not remnants of more extensive tissues connecting the atria and ventricles at an earlier stage of embryonic life. Similar remarks apply to the appearance of the $\mathrm{S}$-A node at a later date $(100 \mathrm{~mm}$.).

\section{Summary AND CONCLUSIONS}

1. The specialized muscle fibres comprising the conducting system of the hearts of mammals and birds include S-A node, A-V node, A-V bundle and its two limbs, and terminal ventricular subendocardial and penetrating Purkinje fibres. Added to these in the bird's heart are the atrial subendocardial and penetrating Purkinje fibres, the right A-V ring, and the special branch of the right limb of the A-V bundle to the muscular $\mathrm{A}-\mathrm{V}$ valve.

While the main topography of this system is similar in mammals and birds, differences are correlated with functional requirements.

2. The specialized cardiac conducting system of these homoiothermal vertebrates is not a remnant of more extensive tissues of similar structure in lower vertebrate hearts. It is a neomorphic development, associated with the more rapid rate of the heart (more rapid, in proportion to its size). Ontogenetic development of these structures in the mammal (calf) supports this view.

3. In a lowly generalized vertebrate heart (Salamander) no specialized tissue is present. The sequences of the cardiac cycle are similar to those of the higher vertebrates. Each chamber has its own intrinsic rhythmic rate, the reason for which is not clearly established. The glycogen content of the musculature of the heart of the frog, which is also devoid of specialized tissue, is inversely proportional to the intrinsic rhythmic rates of the several chambers. 
4. In mammals and birds, parallel evolution of the specialized conducting system has taken place; small differences in the topography of the specialized conducting fibres in closely allied species, or in different animals of the same species, may be attributed to variation.

\section{REFERENCES}

In this brief survey no attempt is made to incorporate all revelant bibliographic references, which will be found in the works cited below.

Blair, D. M., and Davies, F. (1935). J. Anat., Lond., 69, 303.

Davies, F. (1930). Ibid., 64, 129 and 319; and (1931), 65, 339

and E. T. B. Francis (1941). Phil. Trans. Roy. Soc., B,, No. 578, 231, 99.

- (1941). J. Physiol., 100, 329. 